\title{
Reducing CIED Lead Dislodgements: Faithful Alignment to Small Things
}

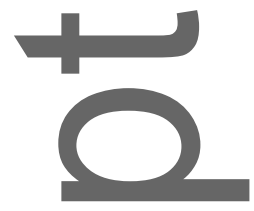

\author{
Frank Pelosi, Jr., MD, FACC, FHRS
}

Running Title: Standardizing surgical techniques to reduce CIED lead dislodgements

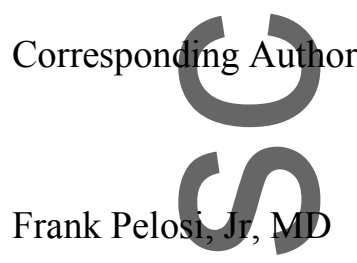

Associate Professor of Medicine

University of Michigan Medical School

1500 East Medical Center Drive

Ann Arbor, Michigan 48109

USA

$734-232-0 \mathrm{r12}$

fpelosi@med.umich.edu

Be faithful in small things because it is in them that your strength lies.

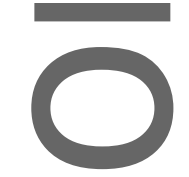

-Saint Mother Teresa of Calcutta(1)

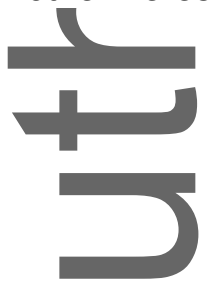

This is th auth $\mathrm{r}$ manuscript accepted for publication and has undergone full peer review but has not been throug - copyediting, typesetting, pagination and proofreading process, which may lead to differences between this version and the Version of Record. Please cite this article as doi: 10.1111/pace.13545.

This article is protected by copyright. All rights reserved. 
Vigilance is critical for the perioperative and long-term success implantation of a cardiac implantable electronic devices (CIED). Often, an extra minute of attention can save hours and prevent complications with both clinical and economic consequences. Attention to the early, seemingly minor, details of the implant procedure can lead to a series of events resulting in either an excellent, efficient outcome or a prolonged procedure that becomes more complex than it was ever intended. Many CIED-related complications are now tracked as institutional quality markers. The US Center for Medicare and Medicaid Services' Physician Quality Report System requires reporting of Medicare and Medicaid patients that experience defibrillator-related procedural complications that require revision and any CIED infections within 180 days of implant.(2) Failure to meet these benchmarks can result in economic penalties for hospitals and clinicians. Unlike the more extensive benchmarks in plance for cardiology, the development and compliance of electrophysiology quality measures remain in their infahcy. Procedural benchmarks are even more difficult to maintain because of rapidly evolving technologies and procedural techniques. At an institutional level, the mitigation of these complications is further challenged as electrophysiology clinicians in larger groups have trained with differing surgical styles, resulting in procedural variability. One of the most important CIED implant complication is lead dislodgement (LD). Several studies have reported that LD and resulting lead revision are associated with infection, prolonged hospital stays and increased mortality. (3-5) Other complications have included valve dysfunction, ventricular arrhythmias, cardiac perforation and death. (6-9)This month's issue of PACE highlights the importance of both vigilance and the provider alignment in reducing this important and often preventable complication.

Afzal and colleagues report the usefulness of a prospective standardized intraoperative protocol to asses lead stability in patients receiving pacemakers, ICDs and CRT devices. [ref] The implanters used intraoperative provocative measures such as manual lead manipulation, and pacing threshold determination during deep inspiration or cough as well as sensing changes over time. 
Change in capture threshold during deep breathing or coughing was assessed in all three leads, while a provocative manual manipulation maneuver was performed in the right atrial lead. The protocol was initiated in consecutive patients for 2 months, then suspended or 2 months to control for increased vigilance of $L D$ and then restarted. The single-site study population was quite large: 2361 patients in the intervention group and 4292 in the control group. The control groups were procedures performed before the initial protocol initiation and the 2-month period when the protocol was suspended. All implanted right atrial (RA) and right ventricular (RV) leads were active fixation leads and more quadripolar coronary sinus (CS) leads were implanted in the intervention group.

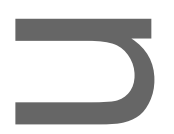

The standardized LD reduction protocol resulted in a $60 \%$ reduction in lead dislodgement. Reduction in LD was observed in all three types of leads; with the biggest reduction in the CS lead, followed by the RA lead then the RV lead. Importantly, reduction in LD was observed even when somewhat aggressive provocative manipulation was performed on the RA lead. The reductions in CS lead dislodgment may be due to the increased use of quadripolar leads, which can be placed in a more stable, distal positon while pacing from the more proximal electrodes. Due to large reductions in CS and RA lead LD, the distribution of right ventricular LD increased from $35 \%$ of all dislodgements at baseline to $50 \%$ after the LD reduction protocol. Unlike prior reports, multivariate analysis showed no independent predictions of LD.

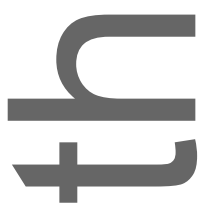

The study does have limitations. Prompting a sedated patient to cough or breathe deeply could make some of the provocative maneuvers difficult. No provocative manipulation was attempted in the RV lead, which could be used to identify potential LD. At follow-up, patients were referred for lead revision on clinical grounds without similar standardized criteria that was used at implant. 
Assessment of threshold with deep breathing or coughing was not assessed at follow-up visits. The authors did not mention whether the two-month suspension of the LD protocol resulted in an increase in LD as compared to the pre-protocol group. Since the biggest reduction in LD occurred in the CS leadgroup, one can question if advancement in lead technology had a greater impact with this lead rather than any provocative measures.

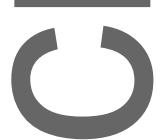

So how is this study significant and how can we apply this to the single patient? A famous college football coach stressed the importance of alignment when he wrote, "Alignment is a key ingredient to elite performance, because without it, the best strategy in the world cannot be executed." (10) From this, we can conclude that any well-intentioned initiatives to improve quality can fail unless crucial stakeholders are aligned for its success. This requires the necessary structure to drive behavior. One such structure is demonstrated in this report, where clinicians clearly identified and defined the problem, agreed on a simple, standardized corrective action and committed to its consistent execution. Interestingly, the solution was not a vast redesign of their enterprise- it was simply applying a small extra step of vigilance during a critical part of the implant procedure. This vigilance combined with consistent alignment were crucial to achieve these outcomes. One can argue which measure were most impactful, but the most important step was consistent execution across all providers.

Quality metrics in electrophysiology procedures will continue to grow in the coming years. Changing reimbursement models will also increase pressure to minimize repeat procedures, especially due to avoidable complieations. From this study, faithful attention to small steps combined with provider alignment can produce significant results.

This article is protected by copyright. All rights reserved. 


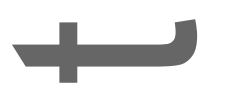

1. Benenate B. In the Heart of the World. Novato, California: The New World Library; 1997.

2. Services USCfMaM. Physician Quality Reporting System Cardiology Preferred Specialty Measure Set 2016 [Available from: https://www.cms.gov/Medicare/Quality-Initiatives-PatientAssessment-Instruments/PQRS/downloads/Cardiology Specialty Measure Set.pdf.

3. Pakarinen S, Oikarinen L, Toivonen L. Short-term implantation-related complications of cardiac rhythm management device therapy: a retrospective single-centre 1-year survey. Europace.

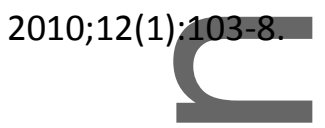

4. Nery PB, Fernandes R, Nair GM, Sumner GL, Ribas CS, Menon SM, et al. Device-related infection among patients with pacemakers and implantable defibrillators: incidence, risk factors, and consequences. Journal of Cardiovascular Electrophysiology. 2010;21(7):786-90.

5. Cheng A, Wang Y, Curtis JP, Varosy PD. Acute lead dislodgements and in-hospital mortality in patients enrolled in the national cardiovascular data registry implantable cardioverter defibrillator registry. Journat of the American College of Cardiology. 2010;56(20):1651-6.

6. Namazi M, Karbasi-Afshar R, Safi M, Serati A. Diaphragmatic stimulation: a case of atrial lead dislodgement and right atrium perforation. Indian Pacing \& Electrophysiology Journal. $2008 ; 8(2): 133-6$.

7. Nair GM, Nair V, Healey JS, Morillo CA. Automatic implantable cardioverter defibrillator lead dislodgement resulting in sudden cardiac death: a case report. Canadian Journal of Cardiology. 2014;30(11):1460.e7-9.

This article is protected by copyright. All rights reserved. 
8. Choudhury PP, Chaturvedi V, Mukhopadhyay S, Yusuf J. Intermittent Right Ventricular Outflow Tract Capture due to Chronic Right Atrial Lead Dislodgement.[Erratum appears in Indian The

Pacing Electrophysiol J. 2014 Jul;14(4):229; PMID: 25067893]. Indian Pacing \& Electrophysiology Journal. 2014;14(4):217-22.

9. Blanco P. ICD Lead Dislodgement and Inappropriate ICD Shocks. CJEM Canadian Journal of Emergency Medical Care. 2016;18(6):489-90.

10. Urban Meyer WC. Above the Line: Lessons in Leadership and Life from a Championship Program. New York, NY: Penguin Books; 2015.

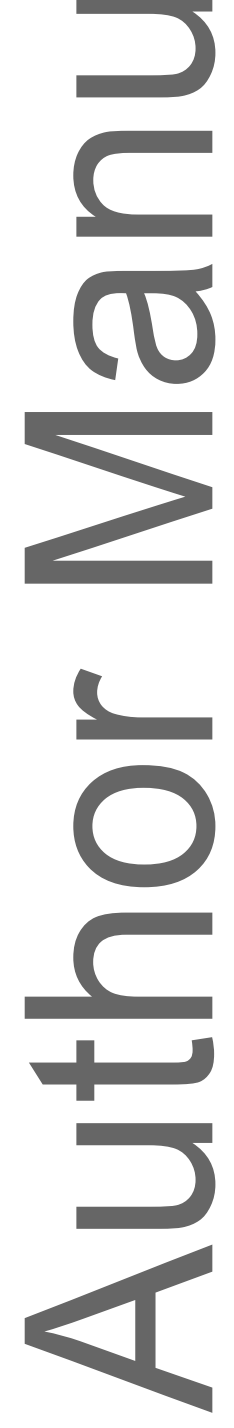

This article is protected by copyright. All rights reserved. 